\title{
Analytical solutions for two-level systems with damping
}

\author{
Christian Brouder \\ Institut de Minéralogie et de Physique des Milieux Condensés, CNRS UMR7590, \\ Universités Paris 6 et 7, IPGP, 140 rue de Lourmel, F-75015 Paris, France.
}

(Dated: May 6, 2019)

\begin{abstract}
A method is proposed to transform any analytic solution of the Bloch equation into an analytic solution of the Landau-Lifshitz-Gilbert equation. This allows for the analytical description of the dynamics of a two level system with damping. This method shows that damping turns the linear Schrödinger equation of a two-level system into a nonlinear Schrödinger equation. As applications, it is shown that damping has a relatively mild influence on self-induced transparency but destroys dynamical localization.
\end{abstract}

Two-level systems have become almost ubiquitous in modern physics. They are found for instance in laser physics, magnetic resonance spectroscopies, quantum computers, quantum teleportation and optoelectronics. The state of a two-level system can be described by an effective moment $\mathbf{M}$ and its dynamics by the equation $\dot{\mathbf{M}}=-\gamma \mathbf{M} \times \mathbf{B}$, where $\gamma$ is the gyromagnetic factor and $\mathbf{B}$ is an external time-dependent field. We follow the standard (and inappropriate) custom of calling $\dot{\mathbf{M}}=-\gamma \mathbf{M} \times \mathbf{B}$ the "Bloch equation".

The main drawback of the Bloch equation is its absence of damping. There are many phenomenological models of damping for two-level systems. When decoherence is small, the system remains in a pure state, the length of $\mathbf{M}$ is constant and damping is taken into account by the so-called Landau-Lifshitz-Gilbert (LLG) equation [1, 2, 3.

$$
\dot{\mathbf{M}}=-\gamma \mathbf{M} \times \mathbf{B}+\frac{\alpha}{M} \mathbf{M} \times \dot{\mathbf{M}},
$$

where $M=|\mathbf{M}|, \dot{\mathbf{M}}=\mathrm{d} \mathbf{M} / \mathrm{d} t$ and $\alpha>0$. It was shown that the LLG equation provides a realistic model of ferromagnetic resonance, micromagnetics, spin-valve dynamics [4], the magnetism of thin films [5] and nanomagnets [6], the dynamics of domain walls in various geometries [7]. Cheng and coll. recently proved that the LLG equations could be derived from a microscopic model [8].

The linear nature of the Bloch equation allowed for the discovery of many analytic solutions. For example, a recent work identified 26 families of solutions that can be expressed in terms of special functions [9] and significant steps toward the general solution were carried out [10]. Even when analytic solutions are not available, powerful analytic approximation methods exist [11, 12, 13].

By contrast, very few solutions of the physically more accurate LLG equation are known. In this paper, we describe a method by which any analytic solution of the Bloch equation can be transformed into an analytic solution of the LLG equation. Similarly, any analytic approximate solution of the Bloch equation is transformed into an analytic approximate solution of the LLG equation. Then, we show that this transformation turns the linear Schrödinger equation for a two-level system into a non linear Schrödinger equation. Finally, we investigate the influence of damping on self-induced transparency and dynamical localization.
We describe now the transformation from the solution of the Bloch equation to the solution of the LLG equation. Consider a solution of the Bloch equation $\dot{\mathbf{M}}(\gamma)=-\gamma \mathbf{M}(\gamma) \times \mathbf{B}$, where the dependence of $M$ on the gyromagnetic factor was written explicitly and where $\mathbf{B}$ is a real function of $t$. Assume now that $\mathbf{M}(\gamma)$ is an analytic function of $\gamma$. This allows us to define $\mathbf{N}=\mathbf{M}(\bar{\gamma})$, where $\bar{\gamma}=\gamma /(1-i \alpha)$. This gives us

$$
\dot{\mathbf{N}}=\dot{\mathbf{M}}(\bar{\gamma})=-\bar{\gamma} \mathbf{M}(\bar{\gamma}) \times \mathbf{B}=-\frac{\gamma}{1-i \alpha} \mathbf{N} \times \mathbf{B}
$$

The equation of motion implies that $M^{2}=\sum_{i} M_{i}^{2}$ does not depend on $t$. We define now

$$
\xi=\frac{N_{x}+i N_{y}}{M+N_{z}}
$$

If we calculate the derivative of $\xi$ with respect to $t$, taking account of the fact that $M$ does not depend on time, we find

$$
\dot{\xi}=\frac{\dot{N}_{x}+i \dot{N}_{y}}{M+N_{z}}-\frac{\left(N_{x}+i N_{y}\right) \dot{N}_{z}}{\left(M+N_{z}\right)^{2}}
$$

If we substitute the equation of motion for $\dot{\mathbf{N}}$, we can check that $\xi$ satisfies

$$
\dot{\xi}=\frac{i \gamma}{2(1-i \alpha)}\left(B^{-} \xi^{2}+2 B_{z} \xi-B^{+}\right)
$$

where $B^{ \pm}=B_{x} \pm i B_{y}$. The complex function $\xi$ is used to define a real vector $\mathbf{M}^{\prime}$ by

$$
\begin{aligned}
& M_{x}^{\prime}=\frac{\xi+\xi^{*}}{|\xi|^{2}+1} M, \\
& M_{y}^{\prime}=-i \frac{\xi-\xi^{*}}{|\xi|^{2}+1} M, \\
& M_{z}^{\prime}=\frac{1-|\xi|^{2}}{|\xi|^{2}+1} M,
\end{aligned}
$$

so that we still have

$$
\xi=\frac{M_{x}^{\prime}+i M_{y}^{\prime}}{M+M_{z}^{\prime}}
$$


but $M_{i}^{\prime}$ are now real. The question is: what is the equation satisfied by $\mathbf{M}^{\prime}$ ? Let us calculate the derivative of $\mathbf{M}^{\prime}$ with respect to $t$. We have

$$
\begin{aligned}
\dot{M}_{x}^{\prime} & =\frac{\dot{\xi}-\dot{\xi}\left(\xi^{*}\right)^{2}+\dot{\xi}^{*}-\dot{\xi}^{*} \xi^{2}}{\left(|\xi|^{2}+1\right)^{2}} M, \\
\dot{M}_{y}^{\prime} & =-i \frac{\dot{\xi}+\dot{\xi}\left(\xi^{*}\right)^{2}-\dot{\xi}^{*}-\dot{\xi}^{*} \xi^{2}}{\left(|\xi|^{2}+1\right)^{2}} M, \\
\dot{M}_{z}^{\prime} & =-2 \frac{\dot{\xi} \xi^{*}+\xi \dot{\xi}^{*}}{\left(|\xi|^{2}+1\right)^{2}} M .
\end{aligned}
$$

If we express $\dot{\xi}$ and $\dot{\xi}^{*}$ through equation (4) and its conjugate, we obtain $\dot{\mathbf{M}}^{\prime}$ in terms of $\xi$ and $\xi^{*}$. If we replace them by equation (5) and its conjugate, we obtain, after a lengthy but straightforward calculation,

$$
\begin{aligned}
\dot{M}_{x}^{\prime}= & -\frac{\gamma}{1+\alpha^{2}}\left(B_{z} M_{y}^{\prime}-B_{y} M_{z}^{\prime}\right)-\frac{\alpha \gamma}{\left(1+\alpha^{2}\right) M} \\
& \times\left(B_{y} M_{x}^{\prime} M_{y}^{\prime}-B_{x} M_{y}^{2}+B_{z} M_{x}^{\prime} M_{z}^{\prime}-B_{x} M_{z}^{\prime 2}\right) \\
\dot{M}_{y}^{\prime}= & -\frac{\gamma}{1+\alpha^{2}}\left(B_{x} M_{z}^{\prime}-B_{z} M_{x}^{\prime}\right)-\frac{\alpha \gamma}{\left(1+\alpha^{2}\right) M} \\
& \times\left(B_{z} M_{y}^{\prime} M_{z}^{\prime}-B_{y} M_{z}^{\prime 2}+B_{x} M_{y}^{\prime} M_{x}^{\prime}-B_{y}{M_{x}^{\prime}}^{2}\right) \\
\dot{M}_{z}^{\prime}= & -\frac{\gamma}{1+\alpha^{2}}\left(B_{y} M_{x}^{\prime}-B_{x} M_{y}^{\prime}\right)-\frac{\alpha \gamma}{\left(1+\alpha^{2}\right) M} \\
& \times\left(B_{x} M_{z}^{\prime} M_{x}^{\prime}-B_{z} M_{x}^{\prime 2}+B_{y} M_{z}^{\prime} M_{y}^{\prime}-B_{z} M_{y}^{\prime 2}\right) .
\end{aligned}
$$

This can be rewritten

$$
\dot{\mathbf{M}}^{\prime}=-\frac{\gamma}{1+\alpha^{2}} \mathbf{M}^{\prime} \times \mathbf{B}-\frac{\gamma \alpha}{\left(1+\alpha^{2}\right) M} \mathbf{M}^{\prime} \times\left(\mathbf{M}^{\prime} \times \mathbf{B}\right) .
$$

We recognize the Landau-Lifshitz equation, in a form equivalent to the LLG equation. To show the equivalence, use the LLG equation to derive $\mathbf{M}^{\prime} \times \dot{\mathbf{M}}^{\prime}=$ $-\gamma \mathbf{M}^{\prime} \times\left(\mathbf{M}^{\prime} \times \mathbf{B}\right)-\alpha M \dot{\mathbf{M}}^{\prime}$, introduce this expression in the LLG equation and solve for $\dot{\mathbf{M}}^{\prime}$. Thus, we have transformed a solution $\mathbf{M}$ of the Bloch equation into a solution $\mathbf{M}^{\prime}$ of the LLG equation (11).

We discuss now the simplest example of this transformation, to show how the method works in practice. We consider a constant external magnetic field along the $z$ axis, i.e. $\mathbf{B}(t)=\left(0,0, B_{0}\right)$. The solution of the Bloch equation is:

$$
\begin{aligned}
& M_{x}=M \sin \theta_{0} \cos \left(\Omega t+\phi_{0}\right) \\
& M_{y}=M \sin \theta_{0} \sin \left(\Omega t+\phi_{0}\right) \\
& M_{z}=M \cos \theta_{0}
\end{aligned}
$$

where $M, \theta_{0}$ and $\phi_{0}$ are constants and $\Omega=\gamma B_{0} . \mathbf{M}$ is obviously an analytic function of $\gamma$ and we can substitute $\gamma /(1-i \alpha)$ for $\gamma$ in $\mathbf{M}$ to define the vector $\mathbf{N}$. This gives us

$$
\xi=\tan \frac{\theta_{0}}{2} \mathrm{e}^{i\left(\Omega^{\prime} t+\phi_{0}\right)} \mathrm{e}^{-\alpha \Omega^{\prime} t}
$$

with $\Omega^{\prime}=\Omega /\left(1+\alpha^{2}\right)$ and we recover the well-known solution of the LLG equation

$$
\begin{aligned}
& M_{x}^{\prime}=M \frac{\sin \theta_{0} \cos \left(\Omega^{\prime} t+\phi_{0}\right)}{\cosh \left(\alpha \Omega^{\prime} t\right)+\cos \theta_{0} \sinh \left(\alpha \Omega^{\prime} t\right)}, \\
& M_{y}^{\prime}=M \frac{\sin \theta_{0} \sin \left(\Omega^{\prime} t+\phi_{0}\right)}{\cosh \left(\alpha \Omega^{\prime} t\right)+\cos \theta_{0} \sinh \left(\alpha \Omega^{\prime} t\right)}, \\
& M_{z}^{\prime}=M \frac{\cos \theta_{0} \cosh \left(\alpha \Omega^{\prime} t\right)+\sinh \left(\alpha \Omega^{\prime} t\right)}{\cosh \left(\alpha \Omega^{\prime} t\right)+\cos \theta_{0} \sinh \left(\alpha \Omega^{\prime} t\right)} .
\end{aligned}
$$

If $\Omega>0$, the equilibrium magnetization is $\mathbf{M}^{\prime}=$ $(0,0, M)$; if $\Omega<0$, it is $\mathbf{M}^{\prime}=(0,0,-M)$. As expected, damping transforms a precession dynamics into a motion towards an equilibrium state.

It is convenient to determine directly the influence of damping on the dynamics of the two-level system described in the Schrödinger or Heisenberg picture. For a two-level system, the Schrödinger equation is

$$
i \hbar \frac{\mathrm{d} \psi}{\mathrm{d} t}=H(t) \psi,
$$

where $\psi$ has two components $\psi_{1}$ and $\psi_{2}$. The Hamiltonian can be written $H(t)=(\hbar \gamma / 2)\left(B_{0}(t)+\right.$ $\left.\sum_{j} B_{j}(t) \sigma_{j}\right)$, where the constant $\gamma$ has been added for later convenience and where $\sigma_{j}$ are the Pauli matrices, so that $\sigma_{a} \sigma_{b}=\delta_{a b}+i \epsilon_{a b c} \sigma_{c}$. Defining $f(t)=$ $\exp \left(-i \gamma \int_{0}^{t} B_{0}(\tau) \mathrm{d} \tau\right)$ and $\psi(t)=f(t) \psi^{\prime}(t)$ turn the Schrödinger equation for $\psi$ into a Schrödinger equation for $\psi^{\prime}$ with the Hamiltonian $H=(\hbar \gamma / 2) \sum_{j} B_{j} \sigma_{j}$. Thus, without loss of generality, we use the latter Hamiltonian.

Following Feynman and coll. [14], the relation between the density matrix $\rho$ (with matrix elements $\rho_{i j}=\psi_{i} \psi_{j}^{*}$ ) and the magnetic moment $\mathbf{M}$ is $M_{x}=\rho_{12}+\rho_{21}, M_{y}=$ $i\left(\rho_{12}-\rho_{21}\right)$ and $M_{z}=\rho_{11}-\rho_{22}$. Thus, we obtain $\rho=$ $(1 / 2)\left(1+\sum_{j} M_{j} \sigma_{j}\right)$ and $|\mathbf{M}|=1$. We see that $\xi=$ $\left(M_{x}+i M_{y}\right) /\left(1+M_{z}\right)=\psi_{2} / \psi_{1}$. If we diagonalize $\rho$ we recover the states $\psi_{1}$ and $\psi_{2}$ up to a phase that cannot be specified easily. Therefore, we shall work with the density matrix. The equation of motion for $\rho$ is

$$
\frac{\mathrm{d} \rho}{\mathrm{d} t}=-\frac{i}{\hbar}[H, \rho] \text {. }
$$

The commutation relations for the Pauli matrices turn this equation into the Bloch equation

$$
\dot{\mathbf{M}}=-\gamma \mathbf{M} \times \mathbf{B}
$$

To turn the Bloch equation into the LLG equation, we just replace $\mathbf{M}$ by $\mathbf{M}^{\prime}$ and $\mathbf{B}$ by $\mathbf{B}-(\alpha / \gamma) \dot{\mathbf{M}}^{\prime}$. If we denote by $\rho^{\prime}$ the density matrix corresponding to $\mathbf{M}^{\prime}$, we find the equation of motion in the presence of damping

$$
\dot{\rho}^{\prime}=-\frac{i}{\hbar}\left[H, \rho^{\prime}\right]+i \alpha\left[\dot{\rho}^{\prime}, \rho^{\prime}\right]
$$

In other words, the damping term of the LLG equation is transformed into a nonlinear term $i \alpha\left[\dot{\rho}^{\prime}, \rho^{\prime}\right]$ in the equation of motion of the density matrix. 
If we replace $\mathbf{B}$ by $\mathbf{B}-(\alpha / \gamma) \dot{\mathbf{M}}^{\prime}$ in the Schrödinger equation itself, we obtain the nonlinear Schrödinger equation

$$
\begin{aligned}
\frac{\mathrm{d} \psi_{1}^{\prime}}{\mathrm{d} t}= & \left(\left(1+2 i \alpha\left|\psi_{2}^{\prime}\right|^{2}\right) B_{3}-i \alpha \psi_{1}\left(\psi_{2}^{\prime}\right)^{*} B_{+}\right) \psi_{1}^{\prime} \\
& +\left(1+i \alpha\left|\psi_{2}^{\prime}\right|^{2}\right) B_{-} \psi_{2}^{\prime}, \\
\frac{\mathrm{d} \psi_{2}^{\prime}}{\mathrm{d} t}= & -\left(\left(1+2 i \alpha\left|\psi_{1}^{\prime}\right|^{2}\right) B_{3}+i \alpha\left(\psi_{1}^{\prime}\right)^{*} \psi_{2}^{\prime} B_{-}\right) \psi_{2}^{\prime} \\
& +\left(1+i \alpha\left|\psi_{1}^{\prime}\right|^{2}\right) B_{+} \psi_{1}^{\prime} .
\end{aligned}
$$

Therefore, our method transforms the analytic solution of a Bloch equation into the analytic solution of a nonlinear Schrödinger equation. We also reach the surprising conclusion that the nonlinear terms in the nonlinear Schrödinger equation can describe the influence of damping. However, this damping does not create decoherence: it transforms $\mathbf{M}$ into $\mathbf{M}^{\prime}$, which is real and satisfies $\left|\mathbf{M}^{\prime}\right|=1$. As a consequence, $\left(\rho^{\prime}\right)^{2}=\rho^{\prime}$ and $\rho^{\prime}$ is the density matrix of a pure state.

We investigate now the effect of damping on two famous nonperturbative effects in two-level systems: selfinduced transparency and dynamical localization.

McCall and Hahn 15] discovered a solution for the hyperbolic secant pulse $\mathbf{B}=(a / \cosh (t / \tau), 0,0)$. We recall that $\int_{-\infty}^{\infty} \mathrm{d} t / \cosh (t / \tau)=\tau \pi$. We consider more generally a spin system submitted to a time-varying magnetic field linearly polarized along $O x: \mathbf{B}=(b(t), 0,0)$, where $b(t)$ is only required to be integrable. At time $t=0$ the spin has the spherical coordinates $\theta_{0}$ and $\phi_{0}$. Let $f(t)=\int_{t_{0}}^{t} \mathrm{~d} \tau b(\tau)$ and $a=\left(1-\xi_{0}\right) /\left(1+\xi_{0}\right)$ with $\xi_{0}=\tan \left(\theta_{0} / 2\right) \mathrm{e}^{i \phi_{0}}$. The solution of the Bloch equation is

$$
\begin{aligned}
& M_{x}(t)=M \frac{1-\rho^{2}}{1+\rho^{2}}, \\
& M_{y}(t)=-2 M \frac{\rho \sin (x(t)+u)}{1+\rho^{2}}, \\
& M_{z}(t)=2 M \frac{\rho \cos (x(t)+u)}{1+\rho^{2}},
\end{aligned}
$$

with $\rho=|a|, a=\rho \mathrm{e}^{i u}$ and $x(t)=\gamma f(t)$. Self-induced transparency occurs when $x(\infty)=2 n \pi$ because, after a long interaction with the external field, the system finds itself in its state at $t_{0}$. In the case of the McCall and Hahn pulse, we find $f(t)=2 a \tau \arctan \left(\tanh \left(\left(t-t_{0}\right) / 2 \tau\right)\right)$, which tends to $a \tau \pi / 2$ for large $t$. Therefore, self-induced transparency occurs when $\gamma a \tau=4 n$ for some integer $n$.

To determine the effect of damping on this phenomenon, we calculate

$$
\xi=\frac{1-a \mathrm{e}^{i \bar{\gamma} f(t)}}{1+a \mathrm{e}^{i \bar{\gamma} f(t)}}
$$

and the corresponding solution of the LLG equation is

$$
\begin{aligned}
& M_{x}^{\prime}(t)=M \frac{\mathrm{e}^{2 \alpha \bar{x}(t)}-\rho^{2}}{\mathrm{e}^{2 \alpha \bar{x}(t)}+\rho^{2}}, \\
& M_{y}^{\prime}(t)=-2 M \frac{\mathrm{e}^{\alpha \bar{x}(t)} \rho \sin (\bar{x}(t)+u)}{\mathrm{e}^{2 \alpha \bar{x}(t)}+\rho^{2}}, \\
& M_{z}^{\prime}(t)=2 M \frac{\mathrm{e}^{\alpha \bar{x}(t)} \rho \cos (\bar{x}(t)+u)}{\mathrm{e}^{2 \alpha \bar{x}(t)}+\rho^{2}},
\end{aligned}
$$

with $\bar{x}(t)=\gamma f(t) /\left(1+\alpha^{2}\right)$. Two effects can be observed. Firstly, the resonance condition is shifted from $x(\infty)=$ $2 n \pi$ to $x(\infty)=2 n \pi\left(1+\alpha^{2}\right)$; secondly, even at resonance the initial state is not fully recovered. For example, if the system is initially in the state $\psi_{1}(0)=1, \psi_{2}(0)=0$, we have $M=1, \rho=1$ and $u=0$ and the final state is, at resonance,

$$
\begin{aligned}
& M_{x}^{\prime}(\infty)=\tanh (\alpha \bar{x}(\infty)) \\
& M_{y}^{\prime}(\infty)=0 \\
& M_{z}^{\prime}(\infty)=\frac{1}{\cosh (\alpha \bar{x}(\infty))} .
\end{aligned}
$$

Thus, it is not possible to recover the initial state because $\alpha \bar{x}(\infty) \neq 0$. Therefore, although the damping of the LLG equation amounts only to a trend towards an equilibrium state and not to a decoherence, it leads to a loss of selfinduced transparency. However, if $\alpha$ is small, this loss is reasonably small and the reader might have the feeling that damping has only a minor effect on the dynamics. The next example shows that this is not the case.

Quantum systems under the influence of a periodic or quasiperiodic external field can exhibit a freezing of the diffusion of its quantum state [16], called dynamical localization. This effect is explained by the Floquet structure of its quantum state. It was analyzed rigorously for a two-level system with $\mathbf{B}=(-f(t), 0, \epsilon)$ by Barata and Cortez [17]. In the case where $f(t)=a \cos \omega t$, they showed that the crucial parameter is $\left\langle q^{2}\right\rangle$, the time average of $q^{2}(t)$, where $q(t)=\exp (i a \gamma \sin (\omega t) / \omega)$. Dynamical localization occurs when $\left\langle q^{2}\right\rangle=J_{0}(2 a \gamma / \omega)$ is zero, i.e. when $\chi=2 a \gamma / \omega$ is a zero of the Bessel function $J_{0}$. In that case, dynamic localization is described as a modulation with a very slow secular frequency $\Omega=2 \gamma^{3} \epsilon^{3} T(\chi) / \omega^{2}+O\left(\epsilon^{4}\right)$, with $T(\chi)=$ $-\sum_{m, n} J_{n}(\chi) J_{n-m}(\chi) J_{m}(\chi) /(m n)$, where $J_{n}$ are Bessel functions and the sum is over all nonzero integers.

In the presence of damping, $\gamma$ is replaced by $\gamma /(1-i \alpha)$ and $\left\langle q^{2}\right\rangle$ can no longer be zero because all zeros of $J_{0}$ are real [18]. Thus, contrary to the case of self-induced transparency, no resonance is strictly possible in the presence of damping. However, a second more dramatic effect occurs. If we take $\chi=2 a \gamma / \omega$ a zero of the Bessel function $J_{0}$, and expand $\left\langle q^{2}\right\rangle$ around $\lambda=\chi /\left(1+\alpha^{2}\right)$, the Bessel expansion[19] yields

$$
\begin{aligned}
\left\langle q^{2}\right\rangle & =J_{0}(\lambda(1+i \alpha)) \\
& =J_{0}(\lambda) J_{0}(i \lambda \alpha)+2 \sum_{k=1}^{\infty}(-1)^{k} J_{k}(\lambda) J_{k}(i \lambda \alpha) .
\end{aligned}
$$


Therefore,

$$
\left\langle q^{2}\right\rangle=-i \chi J_{1}(\chi) \alpha-(\chi / 2)^{2} J_{2}(\chi) \alpha^{2}+O\left(\alpha^{3}\right) .
$$

The off-resonance secular frequency is [17] $\Omega=\epsilon \gamma\left\langle q^{2}\right\rangle$, so that

$$
\Omega=-i \epsilon \gamma \alpha \chi J_{1}(\chi)-\epsilon \gamma \alpha^{2}(\chi / 2)^{2} J_{2}(\chi)+O\left(\alpha^{3}\right) .
$$

The factor $J_{1}(\chi)$ is not zero because the Bessel functions $J_{0}$ and $J_{1}$ have no common zeros [18]. In other words, the secular frequency acquires an imaginary part. Barata analyzed the influence of an imaginary part in detail [20] and showed that the Floquet structure of the wavefunction generates exponentially increasing terms that destroy the unitarity of the quantum evolution. We have shown that the norm of the wavefunction is conserved by the damped dynamics. Thus, the quantum evolution is still unitary and damping destroys the Floquet structure of the wavefunction. In this last example, damping has a dramatic effect on the dynamics of the system. Indeed, experiments confirm that dynamical localization is very sensitive to external perturbations [21].

It was shown in this paper that analytic solutions of the Bloch equations can be transformed into analytic solutions of the LLG equation. The influence of damping leads to interesting results for two-level systems, in particular the transformation of the linear into the non linear Schrödinger equation. The effect of damping was shown to be quite different for self-induced transparency, where it is moderate, and for dynamical localization, where it destroys the structure of the dynamics.

\section{Acknowledgments}

I am very grateful to J. Goulon for discussions on the LLG equation.
[1] L. Landau and E. Lifshitz, Phys. Z. Sowjet 8, 153 (1935).

[2] T. Gilbert, Phys. Rev. 100, 1243 (1955).

[3] T. Gilbert, IEEE Trans. Magn. 40, 3443 (2004).

[4] N. C. Emley, I. N. Krivorotov, O. Ozatay, A. G. F. Garcia, J. C. Sankey, D. C. Ralph, and R. A. Buhrman, Phys. Rev. Lett. 96, 247204 (2006).

[5] P. B. He, X. C. Xie, and W. M. Liu, Phys. Rev. B 72, 172411 (2005).

[6] M. A. Ortigoza, R. A. Klemm, and T. S. Rahman, Phys. Rev. B 72, 174416 (2005).

[7] S. E. Barnes and S. Maekawa, Phys. Rev. Lett. 95, 107204 (2005).

[8] X. Z. Cheng, M. B. A. Jalil, H. K. Lee, and Y. Okabe, Phys. Rev. Lett. 96, 067208 (2006).

[9] V. Bagrov, D. Gitman, M. Baldiotti, and A. Levin, Annalen der Physik 14, 764 (2005).

[10] M. Kobayashi, J. Math. Phys. 45, 486 (2004).

[11] S. Autler and C. Townes, Phys. Rev. 100, 703 (1955).
[12] I. Bialynicki-Birula and Z. Bialynicki-Birula, Phys. Rev. A 14, 1101 (1976).

[13] J. C. A. Barata and W. F. Wreszinski, Phys. Rev. Lett. 84, 2112 (2000).

[14] R. Feynman, F. Vernon, and R. Hellwarth, J. Appl. Phys. 28, 49 (1957).

[15] S. McCall and E. Hahn, Phys. Rev. 183, 457 (1969).

[16] M. Grifoni and P. Hänggi, Phys. Repts. 304, 229 (1998).

[17] J. C. A. Barata and D. A. Cortez, J. Math. Phys. 44, 1937 (2003).

[18] G. Watson, A Treatise on the Theory of Bessel Functions (Cambridge University Press, Cambridge, 1944), 2nd ed.

[19] M. Abramowitz and I. Stegun, Handbook of Mathematical Functions (Dover, New York, 1964), 5th ed.

[20] J. C. A. Barata, Rev. Math. Phys. 12, 25 (2000).

[21] H. Lignier, J. Chabé, D. Delande, J.-C. Garreau, and P. Szriftgiser, Phys. Rev. Lett. 95, 234101 (2005). 